\title{
El sentido del saber en la Escolástica medieval
}

\author{
JaVIER Vergara CIORDIA *
}

\begin{abstract}
RESUMEN
ABSTRACT

La historia del aprendizaje es en parte

The history of Learning is partly

la historia de la cultura. En este the history of Culture. In this article artículo se aborda cómo se entendieron en la Edad Media las relaciones entre el conocimiento y el aprendizaje. En primer lugar se estudia la historia del conocimiento enciclopédico hasta la escolástica medieval; a continuación se analiza la concepción medieval de la historia y del libro, haciendo especial hincapié

en su carácter sacral; el artículo termina analizando la concepción del conocimiento en los enciclopedistas, especialmente en la figura y obra de Vicente de Beauvais, a quien se debe la principal enciclopedia de esta época. we find an approach about the way the relationships between Knowledge and Learning were understood in the Middle Ages. Firstly there is and analysis of the history of encyclopedic knowledge till the scholastic medieval. Then, we find an analysis about the medieval conception of history and of the book, with and special stress in their sacred character. Lastly, the study analyces the conception of Knowledge in the enciclopedists, especially in the figure and work of Vincent of Beauvais, to whom the main encyclopedia of this time is owed.
\end{abstract}

Quoniam multitudo librorum, et temporis brevitas, memoriae quoque labilitas, non patiuntur cuncta, quae scripta sunt, pariter animo comprehendi. Mihi omnium fratrum minimo plurimorum libros assidue revolventi, ac longo tempore

* Dpto. $H_{.}^{a}$ de la Educación UNED. 
studiose legenti, visum est tandem (accedente etiam maiorum meorum consilio) quosdam flores pro modulo ingenii mei electos, ex omnibus fere quos legere potui, sive nostrorum, id est, Catholicorum Doctorum, sive Gentilium, scilicet Philosophorum et poetarum, et ex utrisque Historicorum, in unum corpus voluminis quodam compendio et ordine summatim redigere ${ }^{\text {. }}$.

Este texto, que supone el comienzo de una de las enciclopedias más emblemáticas del enciclopedismo medieval, el Speculum maius de Vicente de Beauvais, 1245-1259, sintetiza en gran medida el sentimiento del hombre escolástico por la cultura. Para el hombre de los siglos XII y XIII, la realidad se sustanciaba en gran medida en términos de conocimiento. Ella es el reflejo de la voluntad divina, la prueba de lo que Dios ha manifestado y permitido. Por eso, conocer, comprender y personalizar la realidad se convierte de suyo en la meta pedagógica del hombre medieval. Veamos cómo los escolásticos asumieron y plantearon este problema desde el plano del conocimiento.

\section{LA RECURRENCIA ENCICLOPEDISTA}

La idea de universalidad o síntesis del saber no era nueva. Podría decirse que el deseo de plenitud intelectual forma parte de la estructura espiritual del hombre y que todas las culturas, en mayor o menor medida, han tenido sus referentes enciclopédicos, variando únicamente su forma de configurarlos y entenderlos. En el mundo clásico, los escritos éticos, políticos y filosóficos de Platón y Aristóteles, de sesgo eminentemente abstractivo y práctico, ya ahondaron en el ideal socrático de acumular en la mente del filósofo todo el saber del cielo y de la tierra, produciendo al respecto sendos y variados ejemplos de literatura preenciclopédica. Aunque serán los escritos de Speusipo (370 a.C.), los Praecepta ad filium de Catón (183 a.C.), los escritos morales de Poseidonio (s. I a.C.) y muy especialmente el Tratado de las nueve disciplinas de Varrón (50 a.C.), junto con los 37 libros de la Historia natural de Plinio (77 d.C.) quienes ostenten el honor de representar de manera más acabada el ideal enciclopédico del mundo greco-romano ${ }^{2}$.

Con el devenir del cristianismo, y más en concreto con los escritos de San Agustín, la dimensión secular del enciclopedismo se tornará de un

\footnotetext{
Vicente DE BEAUVAIS, Libellus apologeticus. Edición fotomecánica de la edición duacense de 1624. Graz-Austria, Akademische Druck-u. Verlagsanstalt, 1964.

2 Cfr. Grimal., P.; «Encyclopédies antiques», en Cahiers d'Histoire Mondiale, 1966, IX, 3, pp. $459-483$
} 
carácter propedéutico que tendrá en las Sagradas Escrituras y en los saberes teológicos su verdadera razón de ser. En De doctrina christiana, el sabio de Hipona ya afirmaba que tenía el deseo de: «reunir en un solo volumen los conocimientos que posee el hombre sobre la naturaleza de las cosas (...) para utilizarlos en beneficio de la Sagrada Escritura» (II, 39, 59). La desmembración del Imperio Romano y la progresiva consolidación de la Iglesia como suprema autoridad moral y doctrinal vino en apoyo de esta idea que primaría sobremanera la preeminencia de una cultura sacral y teológica. El mundo altomedieval conformó la primera etapa de este marco temporal y buscó en De numptiis Philologiae et Mercurii de Marciano Capella (430), en las Institutiones divinarum et saecularium lectionum de Casiodoro (s. vi. ${ }^{\circ}$ ), en las Etimologías de San Isidoro (621) ${ }^{3}$, en el De clericorum institutione de Rabano Mauro (822-836) y en el De divisione naturae de Juan Escoto Erígena (850-875) los mejores referentes enciclopédicos para comprender el mensaje bíblico y teológico ${ }^{4}$.

La Baja Edad Media completaría la segunda etapa de este enciclopedismo teocéntrico de una manera más creativa y fecunda. La progresiva reivindicación de la razón como complemento de la fe en la explicación de las Sagradas Escrituras iba a encontrar en el devenir de los siglos XII y XIII uno de sus periodos más fecundos y emblemáticos. Mucho tuvo que ver en ello la respuesta de Abelardo al problema de los universales, el conocimiento progresivo de la Metafísica, de logica nova y de los libri naturales aristotélicos, junto con el conocimiento de los múltiples escritos de la ciencia greco-árabe. Todo ello posibilitó y consolidó una teoría gnoseológica de vocación enciclopédica y abiertamente convergente; una teoría gnoseológica que, a la vez que entendía la naturaleza como reflejo de la voluntad divina, también la entendía como realidad entitativa y singular que por su propia potencialidad colaboraba en la explicación y sentido de la existencia. Fe y razón, Teología y Ciencia se presentaban ahora como elementos complementarios y como potencialidades diferenciadas de un entendimiento que seguía teniendo como fin último la sabiduría o contemplación divina ${ }^{5}$.

El resultado de este proceso dio lugar a la aparición de múltiples obras enciclopédicas que, si bien no suponían una ruptura brusca con el fin del

\footnotetext{
3 Cfr. FonTAINE, J.; «Isidore de Sevilla et la mutation de l'encyclopédisme antique», en Cahiers d'Histoire Mondiale, 1966, IX, 3, pp. 519-539.

4 Cfr. Gandillac, M.; "Encyclopédies pré-médiévales et médiévales", en Cahiers d'Histoire Mondiale, 1966, IX, 3, pp. 483-519.

5 Cfr. Michaud-Quantin, P.; “Les petites encyclopédies du XIII siècle», en Cahiers d'Histoire Mondiale, 1966, IX, 3, pp. 580-596.
} 
enciclopedismo teocentrico del alto medievo, sí demandaban considerar la multiplicidad y diversidad de la naturaleza en sí misma y por sí misma en tanto que causa coadyuvante de la voluntad divina. La ciencia tradicional parecía pecar ahora de obsolescencia y su estabulación en los viejos límites de las artes liberales se presentaba poco menos que insuficiente para explicar la realidad de los nuevos tiempos. Abrir el conocimiento a la virtualidad de las nuevas ciencias y a los métodos que se oteaban en el horizonte se presentaba como necesidad insoslayable de la que el nuevo enciclopedismo habría de dar cumplida cuenta. Entre los ejemplos más representativos de esta nueva ola enciclopédica pueden citarse las siguientes obras:

- Liber floridus, de Lamberto de San Omerio, 1120.

- De diversis artibus, del monje Theofilus, 1130.

- Imago mundi, de Honorius Augustodinensis, 1130.

- Didascalicon de Studio legendi, de Hugo de San Víctor, 1130.

- Liber exceptionum, de Ricardo de San Víctor, 1140.

- Hortus deliciarum, de la abadesa Herrad, 1180.

- Summa de vitiis et virtutibus, de Raoul Ardent, 1192-1193.

- De naturis rerum, de Alejandro de Neckham, 1203-1213.

- De proprietatibus rerum, de Bartholomeus Anglicus, 1230-1240.

- De naturis rerum, de Tomás de Cantimpré, 1244.

Todas estas obras, amén de un largo elenco de speculum, de los que hablaremos más adelante, influyeron en mayor o menor medida en lo que puede considerarse el ideal de síntesis y universalidad más emblemático y definitorio de la Edad Media: el Speculum maius de Vicente de Beauvais, 1244-1259 ${ }^{6}$.

\section{EL SENTIDO HISTORICISTA}

Para el hombre del medievo, y sobre todo para un escolástico, que lentamente camina del agustinismo al conocimiento del "nuevo Aristóteles", el acceso a la felicidad, a la sabiduría, era un proceso eminentemente intelectual, de progresión continua que, aunque requiriera el auxilio de la fe

6 Cfr. Lemoine, M.; «L'oeuvre encyclopédique de Vincent de Beauvais», en Cahiers d'Histoire Mondiale, 1966, IX, 3, pp. 571-580. 
y de la ciencia, se hallaba ligado inexorablemente a la memoria del pasado, a las res gestae.

Esta idea marcará la personalidad intelectual de muchos escolásticos e impregnará toda la concepción metodológica de sus escritos. El hombre del medievo y más concretamente los enciclopedistas tuvieron un importante espíritu histórico y pedagógico. Fueron grandes compiladores del saber, se esforzaron por ver el hilo conductor de todas las cosas, y se sirvieron del recurso a los florilegios para sistematizar los contenidos, descubrir las relaciones de unas materias con otras y armonizar los elementos más diversos en una perfecta concordancia ${ }^{7}$.

Para los hombres de la Edad Media, y especialmente para los enciclopedistas, la tradición era una ley natural que tenía tanto valor como una ley eclesiástica. Para ellos, nada existe, nada se hace en ningún orden que no tenga su causa, su razón de ser y sus principios esenciales en lo que le precede. Captar el pasado se convirtió en el sentido primordial de su trabajo. Razón por lo que sus obras no alcanzaron la proyección, la creatividad y la altura intelectual de otros medievalistas que buscaron más sistematizar el conocimiento y explicar la realidad. Vicente de Beauvais, haciéndose eco de esta contingencia, confiesa sin rubor que aunque por el método su obra era innovadora, no lo podía ser por las ideas, pues éstas eran antiguas, pertenecían al pasado ${ }^{8}$.

La dimensión historicista del conocimiento medieval no obedecía exclusivamente a un principio lógico de cusalidad. Una razón más profunda alimentó esta posición: la visión escatológica de la existencia. Escolásticos como Hugo y Ricardo de San Víctor defendieron con fuerza que el conocimiento del hombre y de su obra es la historia misma de la salvación humana, marcada por la sucesibilidad de tres momentos determinantes: la creación, el pecado original y la restauración de la naturaleza herida o reencuentro con el Creador. Este último será el punto clave para los escolásticos. Se trataba de buscar en las res gestae los registros didácticos que han ido marcando las formas y modos de acercarnos al conocimiento y unión con Dios.

7 Una de las mejores referencias para estudiar el uso de los florilegios en la escolástica medieval es una serie de artículos de B. L. ULLMAN en los que estudia la utilización de esta técnica por los autores clásicos medievales. Véanse en Classical Philology, XXIII-XXVII (1928-1932). Es particularmente importante el trabajo titulado: «Clasical authors in certain mediaeval florilegia», 27 , 1932.

8 «Quoniam hoc ipsum opus novum quidem est simul \& antiquum, breve quoque pariter \& prolixum. Antiquum certe auctoritate \& materia. Novum vero partium compilacione, \& earum aggregacione". Libellus apologeticus. Op. cit. Cap. IV: De utilitate operis \& apologia auctoris, col. 4, a. 
Esta teleología de la historia confirió a la historiografía medieval un marcado carácter sacralizado y religioso que tuvo notable influencia en la determinación de las fuentes históricas y en su validación. Para el mundo medieval cristiano, y especialmente para los escolásticos esas fuentes se cifraron en siete grados de fiabilidad descendente: Sagradas escrituras, decretos papales, cánones, legislación conciliar, escritos de los Padres de la Iglesia, escritores cristianos no canonizados, y autores paganos. Criterios referenciales que, a medida que se desciende en su escala, disminuyen su certeza y su historicidad para dar entrada a una mayor discriminación y análisis crítico.

Vicente de Beauvais en el Speculum maius, por ejemplo, no fue un mero e ingenuo recopilador de datos históricos. Conocedor de que sólo es pedagógico la actualización de la verdad histórica, abordó el pasado con la discriminación y el sentido crítico propio de un historiador de su época. Celo que no le eximió de veladas críticas. En ocasiones, se le ha achacado presentar contradicciones o sacar sus fuentes de contexto, opinión que previene advirtiendo al lector que sus citas no tienen el mismo valor ni grado de veracidad, por eso las dividió en importantes, regulares, insignificantes y carentes de autenticidad ${ }^{9}$. Igualmente se le achacan errores e imprecisiones considerables en el modo de citar. Críticas que, aunque reflejan una realidad cierta, no revelan falta de escrupulosidad sino el modo de citar propio de la Edad Media. Su fino espíritu crítico, por ejemplo, le llevó a lamentar que trabajos de San Agustín, San Jerónimo o San Juan Crisóstomo hubiesen sido, por ignorancia, atribuidos a San Gregorio y San Isidoro o viceversa ${ }^{10}$; del mismo modo, muchos errores de transcripción - muy comunes en el mundo medieval - los explicó diciendo que fueron debidos a la diversidad de escribanos y amanuenses que trabajaron en su obra, aunque matizó que las imprecisiones no alteraban el sentido y la idea originaria de los autores citados ${ }^{11}$.

\footnotetext{
9 «Ex praedictis, itaque patet non omnia, quae in hoc opere continentur, paris auctoritatis esse, sed quaedam in eis supremum auctoritatis locum tenere, quaedam vero medium, \& quaedam infimum, quaedam vero nullum». Libellus apologeticus, cap. XI: De impari auctoritate eorum, que excerpta sunt, col. 9, c.

io "Adistud ipsum provocavit me plurimun falsitas, vel ambiguitas quaterniorum, in quibus auctoritates Sanctorum adeo plerumque mendaciter a scriptoribus, vel notariis intitulabantur cum scribebantur, ut que esset sententia, vel cuius auctoris esset omnino nesciretur. Dum, verbi gratia; quae Augustini, vel Hieronymi erat, ascribebatur Ambrosio, vel Gregorio, vel Isidoro, vel econtra". Libellus apologeticus, cap. I: De causa suscepti operis \& eius materia, col. 1, c-d.

11 «Intentus, non omnia manu propria, sed pleraque per manus notariorum abbreviavi, ut potui'». Libellus apologeticus, cap. III: De modo agendi \& titulo libri, col. 3, c.
} 
Esta preocupación crítica trascendía con mucho el puro y simple conocimiento del pasado y pretendía hacer de la historia un saber práctico y ejemplar que facilitase el camino hacia la sabiduría o contemplación divina. Con esta instrumentalización, los escolásticos abordaron uno de los problemas historiográficos de mayor complejidad y calado: los modos de interpretar el pasado. Ya en el siglo XII, Hugo de San Víctor había planteado esta cuestión, sustanciando el problema en la combinación de una interpretación literal, alegórica y tropológica. En el siglo XIII, hombres como Guiberto Noguent, Hugo de Saint-Cher y Tomás de Aquino añadieron una cuarta posibilidad: la anagónica o mística, pero ésta apenas tuvo repercusión. La mayoría de los enciclopedistas apostaron por el realismo del pasado, sin desdeñar por ello la dimensión alegórica y tropológica. Vicente de Beauvais fue un fiel exponente de esta postura ${ }^{12}$.

\section{LA SACRALIZACIÓN DEL LIBRO}

En el marco de estas consideraciones historiográficas, el libro se presentaba como el depósito material en el que se almacenarían los registros de la historia. El lugar donde se sintetizarían las res gestae y donde se debía actualizar y proyectar la información conquistada y revelada de la creación. El libro adquiría con ello un sentido escatológico y santificador de primer orden, pues no sólo se consideraba el primer instrumento material de la ciencia, también se consideró un tesoro irrenunciable para la otra vida, una vía de salud capital para el alma y un camino insoslayable de perfeccionamiento.

Consideraciones de este talante dieron pie a una proliferación notable de bibliotecas, a la copia de obras antiguas, códices y manuscritos, a la consolidación de la figura prófesional del monje amanuense y a diferentes disposiciones en favor del libro. En el derecho particular dominicano especial significación cobraba el punto 32 del Capítulo de 1236, donde se prescribía la responsabilidad inexcusable que cada convento tenía en la compra y copia de libros, códices y manuscritos, matizando

\footnotetext{
12 Esta idea la defiende con vehemencia en el capítulo XV del De eruditione Filiorum regalium, donde retoma los modos de interpretar el pasado y afirma al respecto: «Por tanto todas las artes se subordinan a la divina sabiduría y la ciencia inferior, rectamente ordenada, conduce a la superior. Bajo el sentido que media entre las palabras y las cosas, se contiene la historia, a la que se subordinan tres ciencias: la gramática, la dialéctica y la retórica. Bajo el sentido que media entre las cosas y los hechos místicos, se contiene la alegoría. Y bajo el sentido que media entre las cosas y los hechos místicos que han de suceder, se contiene la tropología. A estas dos se subordinan la aritmética, la música, la geometría, la astronomía y la física». Edición Rostok, 1477.
} 
que los libros podrían acompañar a los lectores en caso de traslado a otro convento, con la grave obligación de a su muerte devolverlos a la provincia de origen. En la misma línea, el Capítulo de Bolonia de 1252, recomendó prestar las obras a los conventos que careciesen de ellas, prohibiendo venderlas, salvo para la compra de otras mejores. Medidas que trascendían con mucho el puro interés especulativo o práctico del saber. Para un dominico y para un escolástico en general el conocimiento de los libros y la aprehensión de su ciencia acercaban al ser, santificaban y conferían una forma indeleble en la medida que el saber sólo se desvanece en cuanto a la forma e imperfección, pero no en cuanto a su sustancia. Afirmación que los escolásticos refrendarán afirmando con san Jerónimo: «Aprendamos en la tierra la ciencia de aquello que perseverará con nosotros en el cielo» ${ }^{13}$.

Desde un punto de vista operativo, esta visión santificadora del conocimiento se presentaba como una tarea ardua y compleja por la imposibilidad de asimilar en los estrechos límites de la memoria física, en la escasez temporal de la existencia, y en el espacio material de una obra enciclopédica la gran cantidad de información que la historia de la cultura había producido y que quizá el dinamismo intelectual de los siglos XII y XIII dificultaba todavía más.

Hugo de San Víctor ya vislumbraba esta dificultad en su Didascalicon de studio legendi (1120), y ofrecía la virtualidad del resumen como solución al problema. «Nosotros — decía- debemos abstraer y hacer resúmenes de todo lo que aprendamos, para ser guardado en la pequeña caja de la memoria" ${ }^{14}$. Con la misma intención, Ricardo de San Víctor publicaba en el último tercio del siglo xII un libro cuyo título era lo suficientemente significativo para comprender la necesidad del resumen: el Liber exceptionum. Alentado por las ideas de estos autores y urgido por la necesidad de responder a un problema que empezaba a ser grave, Vicente de Beauvais comenzó su Speculum maius afirmando: "La abundancia de libros, la escasez de tiempo y la fragilidad de la memoria no permiten retener por igual lo mucho que se ha escrito. Por eso a mí, el más humilde de los hermanos, rodeado siempre de libros de muchos autores, y que paso mucho tiempo leyéndolos, aconsejado además por mis superiores, me pareció oportuno compendiar en una sola obra, ordenada y organizada de un modo determinado, un florilegio, elegido a mi criterio,

\footnotetext{
13 San Jerónimo, Epistolae, LIII, 9.

14 Hugo de San Víctor, Didascalicon de studio legendi, 3,11. El texto latino fue editado por Buttimer, C.H. en 1939. Una traducción inglesa fue realizada en 1961 por J. Taylor.
} 
de casi todos los libros que he podido leer, tanto de autores nuestros, es decir, doctores católicos, como de los gentiles (filósofos o poetas), o historiadores de unos y de otros. ${ }^{15}$.

La tarea indudablemente no resultaba sencilla. Hugo, Ricardo de San Víctor y un elenco amplio de autores lo habían intentado antes, pero sus trabajos quedaron en meros resúmenes escuetos de un saber científico al que el dinamismo de la nueva centuria demandaba mayor amplitud y extensión. La empresa indudablemente era difícil, suponía todo un reto a los planteamientos mnemónicos de la época, y sólo una apuesta firme por la selección y presentación óptima de la información podían hacer viable la posibilidad de un conocimiento total. Esto es el Speculum maius: un esfuerzo mnemotécnico sin igual; un intento bibliográfico que impuso al libro exigencias técnicas insoslayables: introducción, índice, tabulación temática por libros y capítulos, concordancias, resúmenes analíticos, toponímicos, etc. Exigencias instrumentales que Vicente de Beauvais supo manejar con la brillantez y extensión propias de un maestro, virtud que a la postre le convirtieron en uno de los enciclopedista más reputados y citados del mundo medieval ${ }^{16}$.

\section{LA REFRACCIÓN O TEORÍA DEL ESPEJO}

La idea de los escolásticos medievales de proporcionar a sus coetáneos y a la historia de la cultura una síntesis extensa y profunda del saber estaba muy lejos de una finalidad intelectualista o dialéctica. Su propósito, encerraba un sentido más profundo, práctico y vital. Se trataba de alcanzar con la información la meta anhelada de todo hombre: la felicidad. Finalidad que implicaba una eminente dimensión gnoseológica al otorgar al conocimiento la más alta de las consideraciones: erigirlo como iter fundamental de acceso al ser, de acceso a la sabiduría, o lo que es lo mismo: el conocimiento se presenta en el escolasticismo como vía y camino insoslayable de acceso a la felicidad; es el ornato más bello del alma.

Esta idea guarda estrecha relación con el término speculum que acompañó a muchas obras medievales. El término speculum encierra

15 Libellus apologeticus, cap. I: De causa suscepti operis \& eius materia, col, 1, a-b.

16 El Speculum en su vertiente instrumental y técnica es estudiado por SMITS, E.R., «Vincent of Beauvais: a note background of the Speculum», en Vincent of Beauvais and Alexander the Great, Studies on the Speculum Maius and its Translations into Medieval Vernaculars, Groningen, E. Forsten, 1986, pp. 1-11. 
todo un género literario que simbolizó el modo de pensar de una parte considerable de la cultura medieval. Para los que así pensaban, y no eran pocos, la teoría de la refracción consistía en plasmar el proceso didáctico del conocimiento refractario de manera sistemática y casi completa, a través de la simbología que encierra la teoría de la visión catóptrica o conocimiento indirecto de realidades superiores mediante procesos de refracción ${ }^{17}$. Los escolásticos, que conocían muy bien los textos sagrados, no ignorarón que fue San Pablo, en la primera Carta a los corintios, quien de modo explícito formuló la pertinencia didáctica de la refracción al afirmar: "vemos ahora mediante un espejo, confusamente; entonces veremos cara a cara» $\left(1\right.$. Cor.13,12) ${ }^{18}$. Aunque serán los neoplatónicos y los Padres de la Iglesia, especialmente San Gregorio de Nisa y San Agustín, los que completarán los principios gnoseológicos de un género literario que tendrá en la teoría de la refracción la base del conocimiento intelectual.

Un elenco amplio de obras refrendará estos principios. La serie se inicia con el Speculum ecclesiae, de Honorius Augustodinensis, escrito a comienzos del siglo XII, y con el Speculum virginum, anónimo aunque atribuido con frecuencia a Conrad de Hirsau, aparecido en el año 1127. Con anterioridad a estas obras, el término speculum aparecía aisladamente sin constituir un género literario. Habrá que esperar al dinamismo gnoseológico y pedagógico del siglo xII para que la refracción adquiera carta de naturaleza didáctica con entidad propia y se aplique con profusión a esferas ético-religiosas, generando los libros-espejo más diversos. Entre los numerosos títulos, podemos señalar:

- Speculum fidei, de Guillaume de Saint Thierry, 1140-1144.

- Speculum caritatis, de Aelred de Rievaulx, 1142-1143.

- Speculum stultorum, de Nigel de Longchamps, 1180.

- Speculum regum, de Godofredo de Viterbo, 1183.

- Speculum poenitentiae, de Renier de Saint-Laurent, c. 1180.

- Speculum universale, de Raoul Ardent, c. 1199.

- Speculum monachorum, de Arnaldo de Boheríes, c. 1200.

17 Cfr. Grabes, H.; Speculum, Mirror und Looking-Glass, Kontinuität und originalität der Spiegelnaetapher in den Buchtiteln des Mittelalters und der englischen Literatur des 13. bis 17. Jahrhunderts. Tübiengen, 1973.

18 Sobre la teoría de la refracción como forma de conocimiento en la antigüedad véase Hugede, N.; La métaphore du miroir dans les épitres de saint Paul aux Corinthiens. Neuchâtel, 1957. 
- Speculum spiritalis amicitiae, de Thomas de Frakaham, inic. s. XIII.

- Speculum humilitatis, de Thomas de Frakaham, inic. s. XIII.

- Speculum futurorum temporum, de Gébénon de Eberbach, c.1220.

- Speculum novitii, de Etienne de Sally, 1234.

- Speculum ecclesiae, de San Edmundo de Abigton, 1240.

- Speculum ecclesiae sive expositio missae, de Hugo Saint-Cher, 1248.

En todas esta obras, una constante aparece como denominador común: la referencia gnoseológica a Agustín de Hipona, tamizada en distinto grado y matiz por la impronta filosófica de San Anselmo, Guillermo de Champeaux, Bernardo de Claraval, Abelardo y muy especialmente por Hugo y Ricardo de San Víctor. Todos estos autores supieron reinterpretar al sabio de Hipona de una manera lo suficientemente creativa y fecunda como para hacer de la dimensión secular de la naturaleza una de las vías insoslayables de acceso a la sabiduría. El salto cualitativo con relación al mundo altomedieval era importante. Ahora, la naturaleza, en su múltiple diversidad, empezaba tímidamente a cobrar sentido propio, entidad por sí misma y una notable finalidad didáctica: se presentaba como iter necesario para captar y entender el reflejo de la divinidad.

La mayoría de los escolásticos del siglo XIII captaron magistralmente este jaque que la historia del conocimiento brindaba a la mente humana y con un posicionamiento gnoseológico de impronta agustiniana y muy cercano al nominalismo moderado de Abelardo, sostuvieron que entre el mundo de las ideas y la realidad sensible o si se prefiere entre el espejo y su reflejo se da una unidad compacta de materia y forma que el entendimiento humano distingue y separa por iluminación y abstracción. Hugo de San Víctor y Vicente de Beauvais fueron fieles exponentes de esta posición. En el capítulo VI del prólogo general del Speculum maius Vicente sostiene al respecto: el mundo por su inmensidad, imita como puede la infinidad de Dios; por su duración indefectible en la sucesión de los seres y de los fenómenos que pasan, nos recuerda su eternidad; y por la multitud y armonía de sus partes, nos representa su hermosura sin mancha y sus perfecciones infinitas ${ }^{19}$. La naturaleza diversa, el mundo creado es, desde esta perspectiva, un efecto de la voluntad divina, un reflejo sin igual de la manifestación de Dios. Explicar ese reflejo o refracción a partir del conoci-

19 «lpse namque mundus spaciositate locorum imitatur pro modulo suo Creatoris immensitatem. Varietate specierum ipsius pulchritudinen, prolixitate temporum eius aeternitatem". Libellus apologeticus, cap. VI: Apologia de naturarerum \& historia temporum, col. 6, b. 
miento de la propia realidad particular constituirá la intención capital de su obra. De ahí que la denominara Speculum. Y si la calificó con el adjetivo maius fue para distinguirla de un trabajo anterior, más reducido (parbulus libellus), llamado Speculum o Imago mundi ${ }^{20}$.

En ese proceso, a los polígrafos medievales les interesaba mostrar la información de la realidad en su máxima extensión. Pensaban, en cierto modo, que el puro hecho de conocer, en tanto que acercamiento a la causa primera, doblega la voluntad y dirige el entendimiento a la contemplación divina. ¿Cómo se produce ese proceso? La respuesta es por el principio de refracción indirecta y la copian literalmente de San Agustín. En primer lugar, recurren al speculum cogitationis ${ }^{21}$ o comprensión de la realidad sensible en su multiplicidad y diversidad. El hombre se encuentra con el espejo del mundo exterior $y$, en la medida que tiene experiencia inductiva de la realidad, descubre, capta, comprende, y contempla la belleza de los elementos de la causa primera reflejados en el speculum.

Esta cogitatio o comprensión no es causada por el mundo exterior. Se produce no tanto por abstracción como por iluminación, por el speculum mentis ${ }^{22}$ o meditación interior. Son los primeros principios que, impresos en el alma en el momento de su creación, iluminan la verdad refractada, la meditan y finalmente la contemplan trascendiendo a ella.

Pero la comprensión del mundo exterior, el conocimiento del yo y la trascendencia de la realidad no son sólo fruto de la acción refractaria del speculum cogitationis y del speculum mentis. El sistema gnoseológico de los polígrafos medievales cuenta todavía con un espejo que, en el orden de la refracción gradual, constituye la autoridad suprema e incontestable de verdad. Es el speculum Scripturae. Al igual que en los casos anteriores, su pertinencia didáctica en el plano gnoseológico es una copia literal del pensamiento agustiniano ${ }^{23}$. Se trata de las Sagradas Escrituras. Estamos ante un espejo nítido, puro; no elaborado por la experiencia humana, ni sujeto a error o desviación. Es la voluntad de Dios objetivada, aquella que se ha mostrado a lo largo de la historia y ha culminado con

20 «Speculum maius appellari decreui (...). Maius autem ad differentiam parvi libelli iamdudum editi, cuius titulus est Speculum, vel imago mundi». Libellus apologeticus, cap. III: De modo agendi \& titulo libri, col. 3, d. El título de Imago mundi es utilizado en torno a 1103-1105 por Honorius augustodunensis con el firme propósito de mostrar la disposición del mundo quasi in speculo. Cfr. FLINT, V.: «Honorius augustodunensis, Imago mundi», Archives d'histoire doctrinale et littéraire du Moyen Âge, 49, 1982, pp. 7-153.

21 Soliloquios, II, 6-10.

22 De Trinitate, XV, 20, 39; XV, 24, 44.

23 Confesiones, XIII, 15. 
Cristo permaneciendo para la eternidad. Su refracción es pura, directa, sin error. Al hombre sólo le queda aprehenderla, hacerla suya.

Indudablemente, en todo este planteamiento, los enciclopedistas medievales, especialmente en el siglo XII y XIII, aunque fueron fieles al postulado credo ut intelligam, intelligo ut credam, adolecieron en no pocos casos - especialmente los Vitorinos y Vicente de Beauvais - de una querencia especulativa e intelectualista que rendía la voluntad al conocimiento del bien. Esta idea, de la que difícilmente pudieron sustraerse, fue consecuencia sobre todo del peso que en su formación tuvo el neoplatonismoagustiniano y de no haber digerido suficientemente el racionalismo arábigo-aristotélico descubierto en el siglo XII. 